\title{
Prototypic force feedback instrument for minimally invasive robotic surgery
}

\author{
Ulrich Seibold, Bernhard Kuebler, and Gerd Hirzinger \\ Institute of Robotics and Mechatronics, DLR (German Aerospace Center)
}

Germany

\section{Introduction}

In recent years the success of the daVinci robotic surgery system (Intuitive Surgical Inc., Sunnyvale, CA, USA) has demonstrated the advantages of a telerobotic approach in minimally invasive surgery (MIS). The worldwide need and acceptance of robotic assistance systems for minimally invasive surgery can be seen with more than 600 sold systems worldwide $^{1}$. Still haptic feedback, important to surgeons who generally rely on the sense of touch in assessing tissue properties, is missing. This is due to the lack of suitable instruments capable of measuring the manipulation forces inside the human body on one hand and the lack of haptic displays for conveying this force information in a comprehensible way to the surgeon on the other hand.

In this chapter we present a prototypic force feedback instrument as well as a surgeon workstation as part of a complete setup for minimally invasive robotic surgery (MIRS). The system serves as technology demonstrator showing the feasibility of integrating advanced manipulator technology, haptic feedback and (semi-) autonomous functionality in the context of MIRS. The system will be used to evaluate the impact and benefit of these technologies and hopefully help to improve the acceptance of advanced MIRS. A selection of surgical applications, notably suturing (anastomosis) of coronary vessels while following the motion of the beating heart (motion compensation), provide the requirements in terms of functionality and performance. In a first step, described in this chapter, components are built and the adherence to the required specifications is assured (objective performance measurement). In a future step the impact and benefit on the selected surgical tasks (subjective performance measurement) will be evaluated by defining relevant experiments and performance metrics. Over the course of the project emphasize is given to generic and modular concepts, as acceptance of MIRS technology will be improved by high usability and good integration into the clinical workflow.

After a short introduction into MIS and MIRS (Section 1.1 and 1.2) the DLR scenario is introduced in Section 2 followed by a selection of related research in Section 3. Main focus lies on an instrument and a surgeon workstation providing haptic feedback, which are presented in Section 4, together with initial results. The chapter is concluded with a critical review of the contributions (Section 5) and closes with an outlook about future research (Section 6).

${ }^{1}$ As of June 30, 2007. North America: 504, Europe: 108, rest of the world: 44 (Intuitive Surgical, 2007).

Source: Medical Robotics, Book edited by Vanja Bozovic, ISBN 978-3-902613-18-9, pp.526, I-Tech Education and Publishing, Vienna, Austria 


\subsection{Minimally Invasive Surgery}

Minimally invasive surgery is an often described and well established operation technique in the so called $11^{\text {st }}$ world country's health care especially for standard procedures like cholecystectomies (gall bladder removals). We, therefore, content ourselves with a very brief description of this method.

In conventional MIS long, slender instruments providing only one functional degree of freedom (DoF), e. g. a grasper at their distal end, are used through small incisions into the patient's skin to access the intra-corporal operation field. For better access to abdominal organs the abdominal wall can be uplifted by insufflating gas (pneumoperitoneum, see Figure 1). The epidermis forms an effective barrier preventing direct view and manual access. Hence, dexterity is heavily restricted and hand eye coordination is disrupted due to the so called chopstick-effect (inversion of movement) and two missing DoF inside the patient caused by the invariant point of incision (fulcrum point), see Figures 1 and 2. Getting used to the handcraft of MIS, therefore, is very protracted and the falsified haptic and tactile feedback is considered to be a hinderance.

\begin{tabular}{|l|l|}
\hline Advantages & Disadvantages \\
\hline - reduced traumatisation & - lost hand-eye coordination \\
- reduced loss of blood & - constricted DoF in instrument handling \\
- reduced risk of wound infection & - 2-D sight, falsification of color representation \\
- reduced postoperative pain & - significantly longer operating time \\
- shorter hospital stays and & - heavily diminished haptic/tactile feedback \\
rehabilitation time & - complex reorientation after instrument changes \\
- faster social reintegration & - expensive and sophisticated equipment necessary \\
- cosmetically favorable results & • long learning curve, high training needs \\
\hline
\end{tabular}

Table 1. Summary of the most important reduced wound healing disorders advantages and disadvantages of MIS; note that almost all advantages affect the patient while almost all disadvantages affect the surgeon
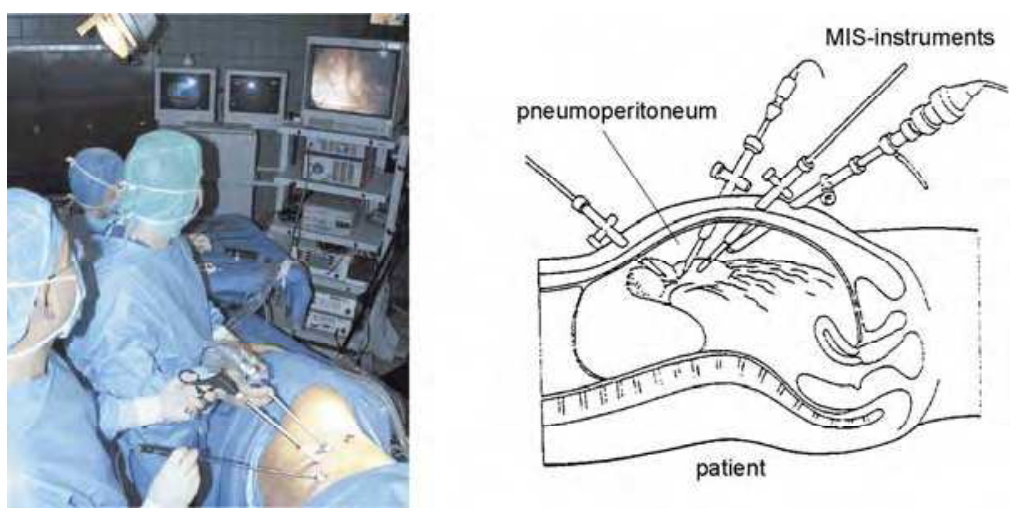

Figure 1. Left: Typical view in an operating room for MIS. In front: surgeons and patient; in the back: technical equipment with monitor. Right: Schematic diagram of instrument insertion in MIS (cholecystectomy) 
Nevertheless, MIS provides fundamental advantages like drastically reduced surgical traumatisation with consecutively shortened time of convalescence and faster social reintegration. The objective is to treat patients with maximum care and, thereby, save costs due to shorter hospital stays and rehabilitation time.

It is evident that almost all advantages are in the interest of the patient while surgeons are burdened with most disadvantages of MIS. A summary of the most important advantages and disadvantages of MIS can be seen in Table 1.

\subsection{Minimally Invasive Robotic Surgery}

As stated above, the handcraft of conventional MIS is very protracted as instrument handling is counterintuitive to open surgery and additional DoF inside the patient are missing. Providing the surgeon with additional manual control elements to operate additional distal DoF was attempted (Tuebingen Scientific Medical, 2007; Frede et al., 2007; Inaki et al., 2007, also see Figure 5), but handling is even less intuitive than in conventional MIS.

Minimally invasive robotic surgery can overcome these drawbacks by using a teleoperated approach: the surgeon comfortably sits at a console controlling the surgical instruments guided by a patient sided surgical robot. Computational support allows for reestablishing hand-eye coordination, motion scaling, indexing (repositioning of the input devices to a comfortable working position while the instruments remain still) and even motion compensation (e. g. breathing motions).
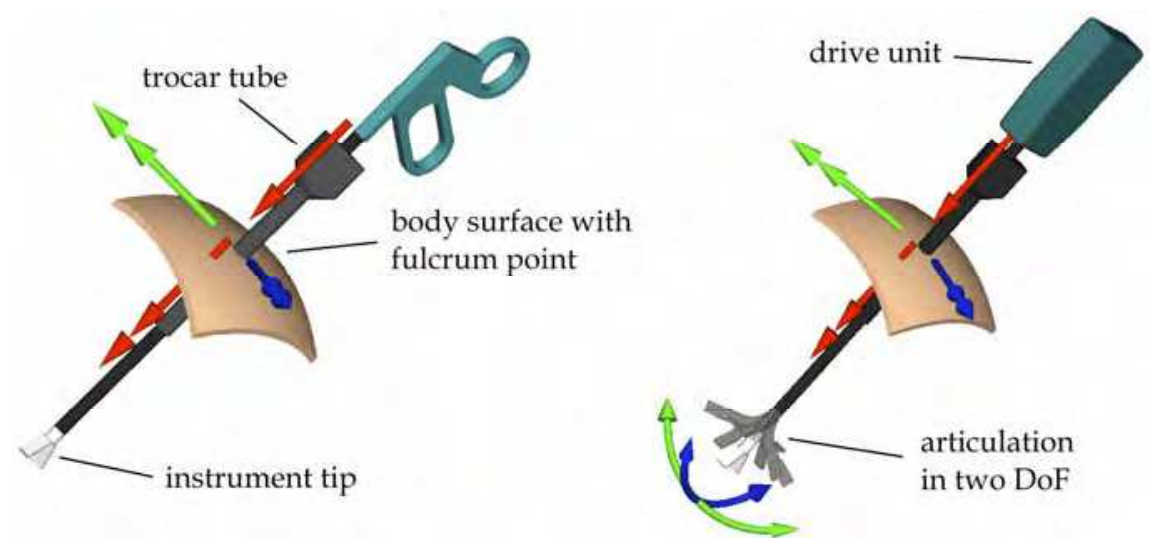

Figure 2. Schematic diagram of DoF in MIS. Rotational DoF indicated with a double arrowhead, translational DoF with a single one. Left: Diagram of the four available DoF in conventional minimally invasive surgery; the instruments are under constraint by the fulcrum point. Right: Diagram of two additional DoF at the distal end of the instrument. Intracorporal DoF are actuated from outside the patient

However, the entirely mechanically decoupled arrangement of surgeon and patient entails a total absence of haptic and tactile feedback, even more so than in conventional MIS. Visual judgement remains the only solution to get an impression of the forces applied to the environment. In fact, the deformation of tissue is patient dependent (Wagner et al., 2002) and even in the hands of experienced surgeons this is not sufficiently reliable and unsatisfying. Considering e. g. knot tying, it is impossible in this way to guarantee a reliable 
tightness of knots (Müller, 2004) because tension of a thread cannot be estimated only by inspecting it visually. Methods of resolution for this problem have been presented by Akinbiyi et al. (Kitagawa et al., 2004; Akinbiyi et al., 2006), but for high immersive surgical purposes this does not seem satisfying. Additionally the presented approaches do not consider gripping forces: Manipulating tissue without gripping force feedback holds the risk of unintentional damage to the tissue.

\begin{tabular}{|l|l|}
\hline \multicolumn{1}{|c|}{ Advantages } & \multicolumn{1}{c|}{ Disadvantages } \\
\hline - reestablishment of hand-eye & - high purchase cost \\
- coordination & - high maintenance cost \\
- intuitive use, short learning curve & - limited number of proven operation \\
- minor fatigue, prolonged concentrated & - types \\
- nork & - cumbersorthy setup time \\
- 3-D vision & - longer operating time \\
- tremor filtering & - usage only as a whole \\
& $\begin{array}{l}\text { - no haptic/ tactile feedback } \\
\text { - necessity of specially trained/educated } \\
\end{array}$ \\
\hline
\end{tabular}

Table 2. Summary of the most important advantages and disadvantages of MIRS

Looking at the presently only commercially available MIRS system (daVinci by Intuitive Surgical, Inc., Sunnyvale, CA, USA) a number of further drawbacks aside from the disadvantages related to the absence of haptic/tactile feedback have to be mentioned.

The still limited number of applications makes it difficult for small and mid-size clinics to have a fast and easy amortization of the considerable purchase and maintenance cost. Setup time of the system and intraoperative instrument changes are still cumbersome which extends the over all operation time even beyond conventional MIS. Obstructive for more universal usage is the fact, that the system can only be used as a whole; e. g. it is not possible to perform a conventional MIS procedure only with the aid of a daVinci camera guidance. The direct access for a surgeon to the patient with the daVinci-slave in position is largely obstructed. Table 2 shows the major advantages and disadvantages of minimally invasive robotic surgery as performed today.

\section{The DLR Minimally Invasive Robotic Surgery Scenario}

The DLR (German Aerospace Center) is currently developing an integrated environment for MIS. The system shown in Figure 3 is divided into a surgeon workstation and a patient-side manipulator setup.

The slave system consists of usually three surgical robots (Figure 3 bottom middle).

Two robots are carrying actuated and sensor integrated surgical instruments (Figure 3 bottom right) which provide additional DoF. The third is used as an automated robotic camera guidance system (Wei et al., 1997; Omote et al., 1999). The robotic arms are lightweight and dimensions are optimized for surgical applications, performing with the highest possible manipulability, dexterity, and accuracy in the important minimally invasive application areas of abdominal and cardiac surgery (Konietschke et al., 2003). Due to the chosen dimensions, this generic manipulator arm is also applicable for urological, 
gynaecological, orthopaedic, and otorhinolaryngological applications. The 7 DoF (kinematic redundancy) design allows for a more flexible operating room setup and facilitates collision avoidance with other manipulators or operating room equipment, as the elbow joint can be reoriented without altering the position and orientation of the robot's end effector.

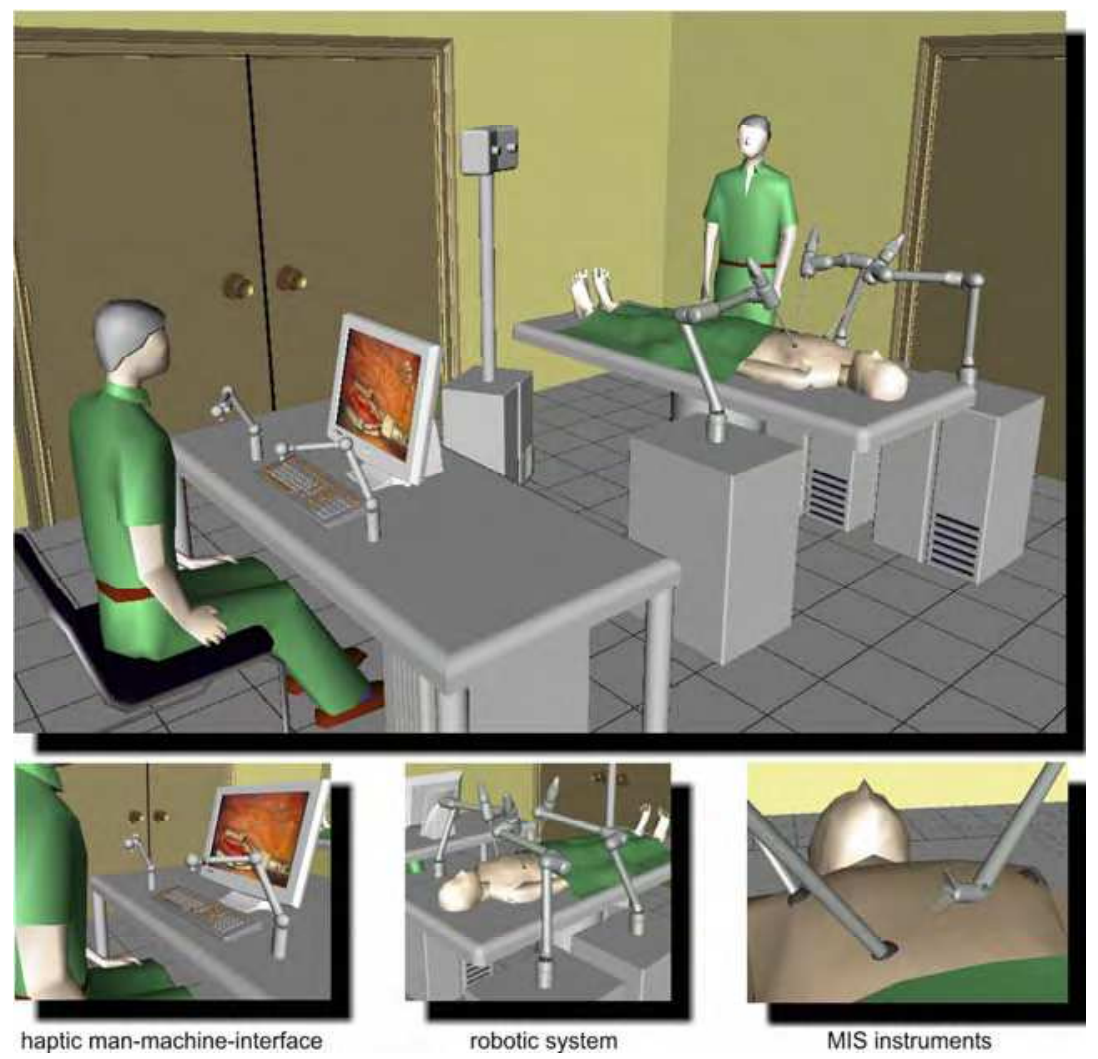

Figure 3. Main components of DLR MIRS system

The master console enables the surgeon to command the instruments and also provides a stereo image of the operation site. Haptic hand controllers not only register the surgeon's hand movements, but also display the intracorporal manipulation forces and torques, including gripping force (Figure 3 bottom left).

The DLR MIRS system will enable surgeons to perform new operation techniques requiring a high degree of manipulability like enteroanastomoses (Müller, 2004) or minimally invasive coronary artery bypass operations on the beating heart (Falk et al., 1999). It will also allow the realization of semi-autonomous functionality such as motion compensation of the beating heart (Ortmaier et al., 2003; Ortmaier, 2003; Nakamura, 2003). Furthermore, the system will include a self-guided robotic camera control system: when advised by the surgeon, the endoscope carrying surgical robot can follow the surgical instruments autonomously. All instruments are equipped with a color marker at the tip. Provided, the same color is not normally present at the operation site, the marker can be segmented from 
the video image with a high degree of robustness (Wei et al., 1997; Omote et al., 1999). Following the segmented positions a trajectory can be calculated which generates the target trajectory for the camera guidance robot. This application has been successfully clinically tested during laparoscopic cholecystectomies.

The MIRS system in development is completed by a preoperative planning and registration tool which allows optimal positioning of ports and robots for the intended operation. The access planning is performed prior to the procedure using the patient specific anatomical data obtained by a medical 3-D imaging modality preoperatively. In the OR, the position of the patient is registered with a 3-D laser scanner, so that the access data can be aligned to the actual patient location very precisely (Konietschke et al., 2004). Intraoperative repositioning of the robots as well as the necessity for placing additional ports intraoperatively can thus be avoided.

The need to perform routine long distance tele-operations is questionable, however, invoking external assistance or a remote expert opinion (teleconsultation) might be realized in the near future. The remote expert will be equipped with a console connected by broadband communication lines to the hospital where the operation takes place. This setup is also especially suited for new surgical training procedures where the remote expert trains the novice surgeon.

This chapter focuses on the development of actuated and sensor integrated forceps as well as on the surgeon input console and on sensory substitution approaches for providing haptic feedback. Details on the development of the surgical robot can be found in (Ortmaier et al., 2006; DLR - German Aerospace Center, Institute of Robotics and Mechatronics, 2007).

\section{State of the Art}

A selection of previous research will be introduced in this section. Improved dexterity and force feedback have shown beneficial effects (see Section 3.1, Section 3.2), however, studies on the effects of force feedback on task performance in laboratory settings are currently limited. Information on the effectiveness for the whole task of surgery does not exist at this point. Exploring the effects of improved dexterity and force feedback simultaneously while using telemanipulators was previously not possible since no setup was available combining intracorporeal degrees of freedom and force sensing capability.

Sensory substitution (auditory and visual instead of haptic feedback) has been shown to be a viable option of providing additional information on the tissue manipulation forces (see Section 3.5). However, the performance of sensory substitution has not yet been directly compared to haptic feedback. If proven effective, sensory substitution could not only be beneficial to the surgical task, but also lead to the development of cheaper surgeon input consoles. This potential is described in Section 4.4 in more detail.

\subsection{The Impact of Dexterity on Surgical Performance}

A comparison of laparoscopic skills performance between standard laparoscopic instruments and two surgical robotic systems was performed by Dakin et al. (Dakin \& Gagner, 2003). In this study all 18 test subjects were skilled laparoscopic surgeons, although only two had prior exposure to telemanipulator systems. They were evaluated on the following tasks using conventional instruments, the daVinci, and Zeus (Computer Motion Inc., USA) robotic surgery system: 
- 'Rope passing': A rope is incrementally passed in both directions using hand-to-hand technique.

- $\quad$ 'Bead drop': In this task beads with a diameter of $6 \mathrm{~mm}$ have to be placed on pegs.

- $\quad$ 'Peanut Task': Cotton balls have to be dropped in cylindrical beakers.

- 'Suturing' was evaluated on a piece of cloth supported by a foam block. Target sizes for the stitches are 1.0, 0.75 , and $0.5 \mathrm{~mm}$ with distances of 5, 3, and $1 \mathrm{~mm}$. Both knot tying and running stitches were evaluated.

The surgeon's work was evaluated for completion time as well as number of errors. None of the telemanipulator systems was faster than conventional technique, however, they seemed to allow slightly higher precision. The average number of errors, e. g. for running sutures using a particular suture material, was highest for manual technique, lowest for daVinci. In all the basic tasks, daVinci outperformed Zeus with regard to speed and showed some increased (although inconsistent) accuracy. It is likely that both the articulated EndoWrist ${ }^{\circledR}$ instruments and the presence of three-dimensional vision in the daVinci system contribute to its superior performance. Only the suturing task is representative for a real surgical task. The other tests, while investigating important task primitives (instrument positioning and grasping) are not sufficient to allow for the prediction of performance during a complex surgical task.

As in conventional laparoscopy, training has an important effect on the performance of the surgeons. The learning curve of six experts (more than 100 laparoscopic cholecystectomies, clinical experience in intracorporal knot tying) and seven novices (less than 50 cholecystectomies, no advanced laparoscopic experience) was evaluated by Hernandez (Hernandez et al., 2004). Using a daVinci surgical system and a small bowel anastomosis model, the study simulated a complex procedure that requires advanced planning and the use of a significant range of skills and entails a longer learning process. The use of a synthetic model makes the experiment reproducible, standardized and allows more objective comparisons. Shape, lumen, and strength of the anastomosis were evaluated as well as time, instrument path length, and the number of movements. No comparison to conventional MIS or manual techniques was included in the study.

Surprisingly, for the daVinci system, results after five training sessions did not significantly vary between novices and experts. The fact that eight out of thirteen subjects (two experts and 6 novices) reached a competent status after only five sessions could mean that the learning process in the daVinci system is shorter than it would be in conventional laparoscopic surgery. The likely conclusion is that the level of immersion and intuitive instrument handling provided by the daVinci system offers advantages for novice surgeons.

\subsection{The Impact of Haptic Feedback on Surgical Performance}

In conventional MIS there is little to no haptic feedback from the operation site. Friction generated within the instrument and between the instrument and the access port greatly exceeds manipulation forces at the operation site. Exerted forces cited in the literature range from $0.3 \mathrm{~N}$ for bypass grafting (Salle et al., 2004) to about $4 \mathrm{~N}$ normal and $50 \mathrm{~N}$ along the instrument shaft in cholecystectomy (Rosen, 2001). This unexpectedly large range is not sufficiently explained and will need to be investigated.

Surgeons are able, through experience, to interpret visual tissue deformation as a measure of external forces and thus compensate for the lack of haptic information. Unfortunately, tissue properties depend on the patient and may also vary with time (Wagner et al., 2002). The 
visual compensation can only be applied when handling soft elastic materials. It is not feasible with bone structures and suture materials due to their rigidity.

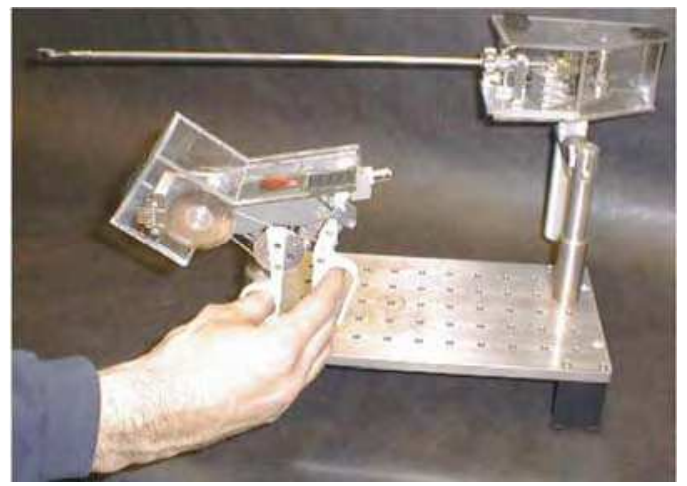

Figure 4. Force controlled gripper designed at the University of Washington (Rosen et al., 1999a)

An experimental sensor integrated gripping instrument was introduced by the BioRobotics Lab, University of Washington, USA (Rosen et al., 1999a). It consists of a conventional laparoscopic instrument which was separated into the gripper/shaft and handle portion (see Figure 4). Both, gripper and handle are actuated by servo drives and controlled in a bilateral force feedback scheme. Using this device several experiments were carried out evaluating the sensitivity and recognition rate while palpating tissue samples.

Test subjects were asked to palpate tissues of varying stiffness by hand, using a conventional laparoscopic grasper and using the sensor integrated instrument. Results were shown as mean square error (MSE) of recognition. As expected, the performance of the human hand defines the upper performance limit and the conventional instrument performed at the lower limit with the sensor integrated instrument performing at a level similar to a gloved hand. Subjective results also showed that the sensor integrated gripper significantly improved the rate of correctly recognized tissue samples. Accurately recognizing the stiffness of tissue will help surgeons to distinguish between different tissue types and assess the health of particular structures.

The role of force feedback in a blunt dissection task was evaluated by R. Wagner (Wagner et al., 2002). During this experiment participants operated on a model of an arterial structure surrounded by tissue. Hand controller and telemanipulator were PHANToM ${ }^{\circledR}$ (SensAble Technologies, Inc., Woburn, MA, USA) haptic hand controllers and forces in the instrument were recorded by a $6 \mathrm{DoF}$ force torque sensor. Tissue was represented by material similar to children's play dough while arteries were represented by weatherstrip and caulking cord. The task was to dissect and expose the structure embedded in the tissue. Individual performance was measured by evaluating the applied force level, number of errors, as well as the rate and precision of the dissection. It was shown that force feedback reduced the magnitude of forces applied on the instrument tip. Higher forces were applied for longer durations when force feedback was not present. The number of errors, defined as punctures and scratches in the artery, was also reduced in the presence of force feedback. The rate of the dissection and the amount of tissue that was disturbed around the dissection area, however, were not significantly influenced by the presence of force feedback. It was hypothesized that at decreasing levels of force feedback the haptic information does not 
constitute a physical constraint anymore but acts as supplemental information. A conscious response is required to take advantage of the available forces.

Participants of this study were novices. Experienced laparoscopic surgeons have developed perceptual and motor skills to deal with the constraints of MIS techniques. The influence of laparoscopic training on the benefit of force feedback needs to be investigated and a more realistic tissue model should be used.

\subsection{Previous Instrument and Sensor Designs}

The foremost example of articulated instruments for MIRS is the EndoWrist ${ }^{\circledR}$ by IntuitiveSurgical ${ }^{\circledR}$ as part of the daVinci robotic surgery system. The instrument design is highly integrated with a diameter of $8 \mathrm{~mm}$, yet extremely rugged and provides two cable driven DoF in addition to the actuation of the functional end (forceps, scissors, and needle holders), thus providing full dexterity inside the body (Guthart \& Salisbury, 2000; Intuitive Surgical, 2007). Recently IntuitiveSurgical ${ }^{\circledR}$ developed even smaller instruments with a diameter of $5 \mathrm{~mm}$, featuring a cable driven spine kinematic for the 2 DoF actuation. However, both instruments are not sensor integrated and so do not allow the measurement of interaction forces and torques.

The Radius Surgical System by Tuebingen Scientific (Braun et al., 2004) closely resembles a conventional hand-held MIS instrument (see Figure 5). As major difference, the functional (distal) end consists of a $2 \mathrm{DoF}$ (pitch-roll) joint, providing full manipulability at the gripper. These two additional degrees of freedom cannot be controlled by the scissor-handle used in conventional instruments. Therefore, an ergonomic handle providing additional functionality was incorporated in the design. The instrument closely resembles a conventional MIS instrument, therefore, providing straight forward integration in the clinical setting at comparably small cost. Surgeons experienced in MIS will adapt their skills readily to the Radius Surgical System. However, the disadvantages of the chop-stick effect and uncomfortable operating posture remains. In addition, demands on the surgeons manual dexterity are greatly increased, as $3 \mathrm{DoF}$ have to be controlled in each hand.
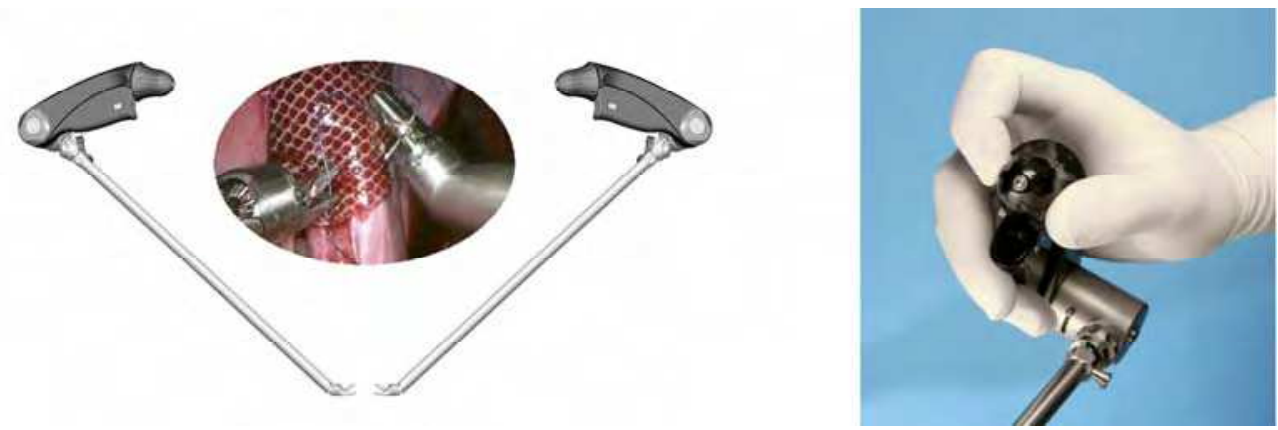

Figure 5. The Radius Surgical System, a novel manually articulated instrument for MIS with ergonomic handle design (Braun et al., 2004)

Full dexterity inside the patient as well as the decoupled determination of grasping force and interaction forces is deemed necessary for an appropriate immersion of the surgeon. Most systems comprising a force sensing modality only focus on one of these three requirements. At the Korean Advanced Institute of Science and Technology (KAIST) a 
telepresence system for microsurgical tasks has been developed. As one of few systems it realizes six DoF force/torque reflection at the master console. Unfortunately, the instruments do not provide additional DoF inside the patient's body (Kwon et al., 1998).

Rosen et al. introduced a teleoperated gripper for MIS (see Figure 4), providing measurement and realistic feedback of endoscopic gripping forces (see Section 3.2). However, this system is limited to the measurement of gripping forces, and so interaction forces cannot be captured (Rosen et al., 1999b; MacFarlane et al., 1999).

Zemiti et al. introduced a force controlled laparoscopic surgical robot without distal force sensing. A standard force sensor was integrated into the trocar outside the patient. The measurement did not deteriorate by friction due to the double-walled design of the trocar (Zemiti et al., 2004). The placement of the sensor requires a very accurate gravity compensation of the results for every position of the Instrument. Additionally, the large distance between instrument tip and sensor entails a deterioration of the force/moment discernibility.

The sensor of the laparoscopic grasper developed by Tholey et al. is integrated in the gripper branches. Forces normal to the gripper branches as well as lateral and longitudinal forces inside the gripper can be registered. Unfortunately, the principle of measurement is not explained in detail but the assembly seems to be prototypic. The instrument has not yet been integrated into a robotic surgery system (Tholey et al., 2004).

Jan Peirs et al. have designed a 3-axis micro optical force sensor. Employing an optical measurement principle, the sensor is inert to electro-magnetic interference. The necessary force range was determined using strain gauges applied onto a needle driver. Future experiments with a laparoscopic system are planned (Peirs et al., 2004).

Several groups (Kitagawa, 2003; Mayer et al., 2004) have equipped daVinci instruments with strain gauges attached to the shaft, close to the joint. This placement does not require the electrical connection to be routed through the joint, which will prolong the life expectancy of the sensor. However, three sets of drive cables for the joint and the gripper are running through the sensor. Therefore, the sensor is subjected to the driving forces which greatly exceed the manipulation loads. The measurement range of the sensor must accommodate for the driving forces, thereby, greatly reducing the sensitivity to the manipulation loads. Compensation is highly difficult since all six cable forces at the sensor location, compensated for friction in the drive mechanism, are required. Additionally, manipulation forces have to be corrected for the joint angle.

\subsection{Surgeon Work Station}

The daVinci robotic surgery system by Intuitive Surgical ${ }^{\circledR}$ (Guthart \& Salisbury, 2000; Intuitive Surgical, 2007) includes a surgeon console. The surgeon sits in a comfortable forward leaning posture at the main console, supported by arm and head rest. By placing the head on the visor, a 3-D image of the surgical field can be seen through a binocular viewer. The hands of the surgeon grasp the master controls, which are situated underneath the visor of the workstation. The placement of the vision system and the master controls convey the impression that the surgeon is looking down at the natural position of the hands and the user has an immersive view of the surgical field. In addition, a light barrier is integrated in the headrest which serves as safety feature. The input controls allow manipulation within a cube with $30 \mathrm{~cm}$ side length, however, do not provide realistic haptic feedback from the operation site. 
Stand alone haptic feedback devices are available from various companies, including the PHANToM ${ }^{\circledR}$ as well as the delta.x and omega.x series (Force Dimension, Inc., Lausanne, Switzerland). None of the above are currently available with 7 active force feedback DoF. Force Dimension has recently added an active grasping DoF and 3 passive (wrist) rotations to the original omega.3, creating the omega.7. An extension to the PHANToM ${ }^{\circledR}$ including a collocated, semitransparent display is available by Reachin Technologies (Inc., Stockholm, Sweden). However, the restricted workspaces of all devices require some indexing or clutch function in order to provide the full range of motion required for various surgical procedures (e. g. exploration of the abdominal cavity or harvesting of the Arteria mammaria). Therefore, a collocated semitransparent display is not usable as the collocation of video image and hand position cannot be guaranteed.

\subsection{Haptic Feedback and Sensory Substitution}

A comparison of the effectiveness of visual and haptic feedback in a laparoscopic grasping procedure was done by Tholey (Tholey et al., 2003). For visual feedback, the participant is shown an isometric view of the grasper and tissue sample (n.b. This description of visual feedback refers only to providing a video feed of the test sample and is not identical to visual augmentation described by Kitagawa below). No grasping force is measured in this setting. This experiment is equivalent to a surgeon observing the visible deformation of manipulated tissue, estimating the strain through experience and assumptions of the tissue properties. Next, for the haptic feedback, the motor torque required to grasp the tissue was recorded and interpreted as a measure for tissue stiffness. In Tholey's experiment, the participants only saw the deformation on the screen or felt the opposing force provided by a PHANToM $^{\circledR}$, while the gripper was closed autonomously to identical angles for every experiment. Several trials were conducted to test the effect of using only the video feed, only force, as well simultaneous video feed as force feedback on rating the stiffness of three samples. The rate of correctly characterizing tissue stiffness was highest for direct exploration by hand, followed by simultaneous video feed and force feedback. The video feed alone produced the lowest rate of success.

The effect of sensory substitution on suture manipulation forces for surgical teleoperation was investigated by Kitagawa (Kitagawa, 2003). Suture forces during knot tying were compared for direct manipulation, conventional MIS technique, and using a telemanipulator setup. The coefficient of variance $(\mathrm{CV})$ between optimal and actually applied suture forces was measured. The results indicate that the manual instrument ties provide a $\mathrm{CV}$ that is more similar to the hand ties than do the robot ties. The hand tie had the lowest CV of all methods, meaning suture forces were consistently applied close to their optimum level. Use of a telemanipulator setup mitigated the difference in skill levels between novice and expert participants. The knot tying procedure using the telemanipulator setup was repeated, providing either only the video image (no feedback) or a visual representation of forces (bargraph or gauge: augmented visual feedback) or an audible tone when the force reached the optimal value (auditory feedback). The provision of any kind of feedback showed significantly improved results. Direct haptic feedback was not provided in this experiment, therefore, a comparison between haptic feedback and sensory substitution feedback was not performed.

Aside from a technical report by Computer Motion, Inc., that only mentioned the possibility of using visual feedback for manipulation forces, no other relevant studies on sensory substitution in MIS were found. 


\section{Description of the Setup}

As shown in Figure 3 and described in Section 2 the surgical instrument shown in Figure 6 is carried by the DLR KineMedic ${ }^{\circledR}$ (Ortmaier et al., 2006; DLR - German Aerospace Center, Institute of Robotics and Mechatronics, 2007) surgical manipulator arm for MIRS applications. The robot is mechanically, electrically, and electronically interconnected to the propulsion unit of the instrument (proximal part, see Section 4.1). At the distal end of the instrument two actuated DoF, a force/torque sensor, and the functional end (e. g. gripper) are located (see Section 4.2 and 4.3). The instrument shaft passes through the hollow axis of the last rotational joint of the KineMedic ${ }^{\circledR}$, so that the instrument can be rotated around its longitudinal axis. With the two additional DoF at the distal end of the instrument full dexterity inside the patient is possible as well as a basic ability to reach behind intracorporal structures. Due to the kinematically redundant design of the KineMedic ${ }^{\circledR}$, its posture can be altered intraoperatively without changing position and orientation of the instrument. Thereby, collisions of robot arms with each other, the patient or other OR equipment can be avoided.

\subsection{Instrument: Propulsion unit}

The instrument is located at the TCP of the KineMedic ${ }^{\circledR}$, therefore, light and compact design especially of the propulsion unit is important not to influence dynamics and performance of the KineMedic ${ }^{\circledR}$ in a negative way. Thus, it is possible to move robot and instrument with the relatively high acceleration necessary to follow the movements of the beating heart.

The propulsion unit provides actuation for the distal joints and gripper, signal conditioning for the force/torque sensor, and contains additional sensors to determine the absolute position of the drive cables. Since the conventional way of sterilization in a hospital is autoclavation and the distal end is in direct patient contact, the instrument has to withstand the according conditions ${ }^{2}$. Therefore, all thermo instable components are placed in the proximal propulsion unit. The distal end (with patient contact) can be detached and autoclaved. The decoupling mechanism allows for relatively simple handling, connectable by technically untrained staff under operation room conditions. The mechanism separates the drive train at the output of the propulsion unit, hence, it is mandatory for satisfactory position control that it is entirely free of backlash and play even after several coupling/decoupling cycles.

\subsection{Instrument: Distal Force Torque Sensor}

To obtain realistic force information a sensor is preferably placed close to the instrument tip, minimizing the errors due to friction between the instrument and the trocar. The sensor should be decoupled from the drive mechanism to prevent the influence of driving forces, backlash, and friction on the sensor's performance (Seibold \& Hirzinger, 2003). A placement between the gripper and joint was selected, as the sensor is only subjected to the gripper actuation force at this location. It is not influenced by the joint actuation forces as in placements proximal to the joint. The gripper actuation force is measured for the calculation of the gripping force, so the force/torque sensor (FTS) output can be compensated for simultaneously. However, the electrical connections to the sensor have to be routed through the joint, requiring highly flexible, isolated, multi-strand wires. This location requires the

\footnotetext{
${ }^{2}$ Hypertensive water steam at up to $156^{\circ} \mathrm{C}$ and up to 2 bar positive pressure for at least $25 \mathrm{~min}$.
} 
sensor to be of roughly cylindrical shape with a preferably central hollow section to accommodate for the gripper drive cable and mechanics.

A Stewart Platform based FTS was chosen for its high stiffness, adaptable properties, annular shape, and scalability. Furthermore, only longitudinal force transducers are required, which facilitates the future application of force transducers other than resistive strain gauges. Analysis and properties of Stewart Platform transducers were presented previously by Sorli et al. (Sorli \& Pastorelli, 1995) who outlined a set of variables

$(R, L, a, \beta, \gamma$, shown in Figure 7 left) sufficiently describing the geometry and, thus, the properties of the sensor. The characteristic matrix $\mathbf{A} \in \mathbb{R}^{(6 \times 6)}$ describing the transformation of link forces to externally applied loads

$$
\left[F_{x}, F_{y}, F_{z}, M_{x}, M_{y}, M_{z}\right]^{T}=\mathbf{A} \cdot\left[F_{1}, F_{2}, F_{3}, F_{4}, F_{5}, F_{6}\right]^{T}
$$

is calculated using the method described by Sorli:

$$
\left[\begin{array}{c}
F_{x} \\
F_{y} \\
F_{z} \\
M_{x} \\
M_{y} \\
M_{z}
\end{array}\right]=\left[\begin{array}{cccccc}
-2 n & 2 n & \sqrt{3} m+n & \sqrt{3} m-n & -\sqrt{3} m+n & -\sqrt{3} m-n \\
-2 m & -2 m & m-\sqrt{3} n & m+\sqrt{3} n & m+\sqrt{3} n & m-\sqrt{3} n \\
-2 q & -2 q & -2 q & -2 q & -2 q & -2 q \\
2 a q & 2 a q & -a q & -a q & -a q & -a q \\
0 & 0 & a q \sqrt{3} & a q \sqrt{3} & -a q \sqrt{3} & -a q \sqrt{3} \\
-2 a n & 2 a n & -2 a n & 2 a n & -2 a n & 2 a n
\end{array}\right] \cdot\left[\begin{array}{c}
F_{1} \\
F_{2} \\
F_{3} \\
F_{4} \\
F_{5} \\
F_{6}
\end{array}\right]
$$

with

$$
\begin{array}{r}
m=\cos (\alpha) \cos (\beta) \\
n=\cos (\alpha) \sin (\beta) \\
q=\sin (\alpha)
\end{array}
$$

To find a sensor geometry that is well conditioned and optimized for the force range expected in a surgical application, the following search method is used. The radius of the base $R$ and the link length $L$ (see Figure 7 left) are determined by the space available in the instrument. For all geometrically valid combinations (non-intersecting links), the parameters $R, L, a, \beta, \gamma$, are used to calculate A. Various sets of maximally expected external loads $\left[F_{x}, F_{y}\right.$, $\left.F_{z}, M_{x}, M_{y}, M_{z}\right]^{T}$ are selected. These sets must contain at least one load in each of the 6 principal directions.

Every member of the external load set is pre-multiplied by $\mathbf{A}^{-1}$, yielding the corresponding set of internal leg forces $\mathbf{J}=\left[F_{1}, F_{2}, F_{3}, F_{4}, F_{5}, F_{6}\right]^{T}$. The conditioning number of the internal leg force set (denoted in Figure 8) is a measure of the isotropy of the sensor structure with respect to the external load set. This, however, is not an isotropy in the classical definition as the external loads in the principal directions need not be equal.

For the load set $F_{x, y, z}=10 \mathrm{~N}, M_{x, y}=150 \mathrm{Nmm}, M_{z}=100 \mathrm{Nmm}$ the following parameters were selected as optimal sensor geometry: $R=4.2 \mathrm{~mm}, L=3.9 \mathrm{~mm}, a=65^{\circ}, \beta=90^{\circ}, \gamma=36^{\circ}$, yielding a conditioning number of 6.3 and a joint separation of $i=1.1 \mathrm{~mm}$.

For a sensor of less than $10 \mathrm{~mm}$ diameter, ball or universal joints normally used in Stewart Platforms are not suitable due to their high complexity of manufacturing and assembly. These are replaced by flexural joints creating a monolithic sensor structure as shown in Figure 7 right (Seibold, 2002). Given appropriate design of flexural hinges and leg cross- 
section, the results of an FEM analysis are in very good agreement with the prediction by the ideal analytical model (Seibold \& Hirzinger, 2003; Lobontiu, 2002).

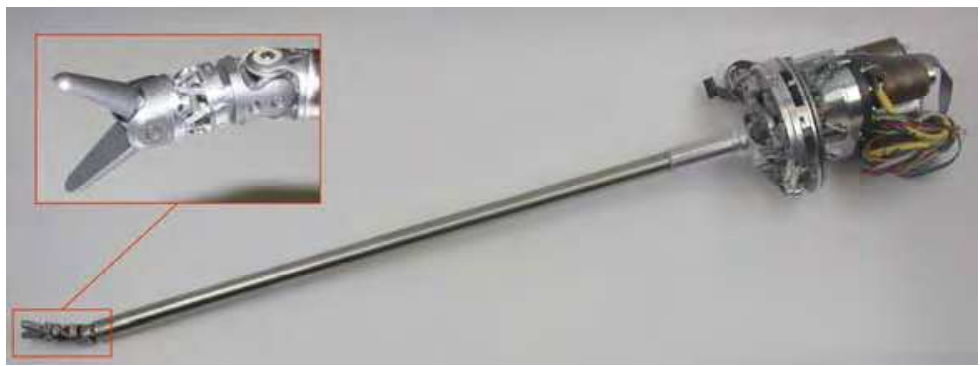

Figure 6. General view of the DLR MIRS instrument and detailed view of its distal end
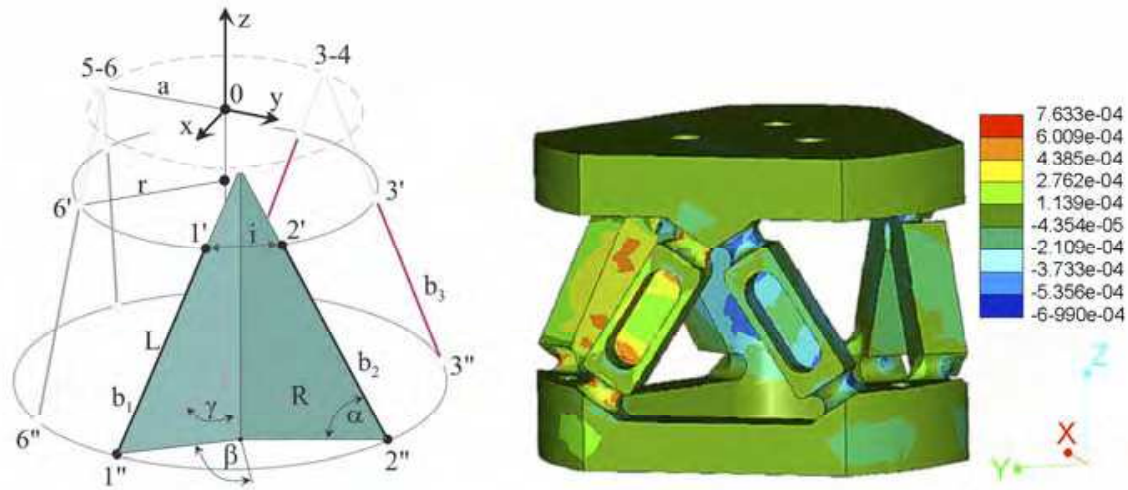

Figure 7. Left: Geometrical parameters of Stewart Platform: Base radius $R$ and platform radius $r$. Right: Average strain on force/torque sensor for load $F_{y}=30 \mathrm{~N}$

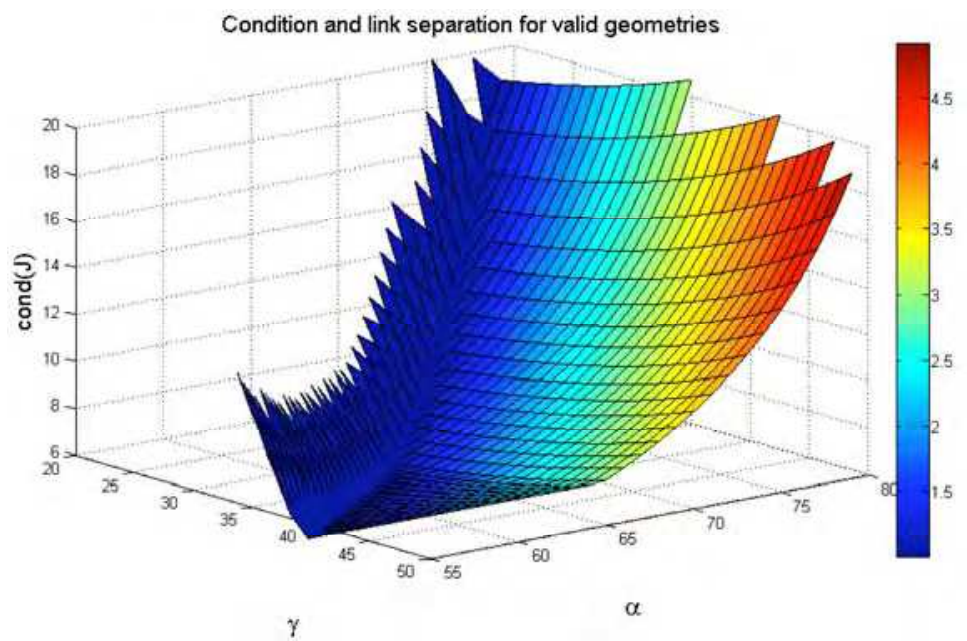

Figure 8. Condition number of internal forces. Colors denote leg separation $i$ at the platform in $\mathrm{mm}$ 
After fabrication and assembly of the strain gauges, the sensor is calibrated using a set of known weights applied in the six principal directions. The sensor is subjected to at least one complete loading/unloading cycle to determine the amount of hysteresis. Results of the calibration for one sensor are shown in Figure 9, with output values for all six load directions being shown in each graph. Output values for the load direction corresponding to the applied external load show an approximately linear unity response, whereas, outputs for all other load directions are expected to remain zero.

An earlier version of the sensor was mounted to the tip of an experimental surgical knife (see Figure 10) and dissection tasks similar to the experiments conducted by Wagner et al. were performed using this setup (Wagner et al., 2002). While the dissection was on average performed $50 \%$ faster by conventional MIS, the damage to the simulated arteries was $20 \%$ lower when using a telemanipulator with force feedback. For detailed discussion of the results see (Deml et al., 2005).

Due to a Quarter-Wheatstone-Bridge configuration and subsequently necessary high amplification the force signal contained 3 bit of noise after 12 bit digitization and was sensible to temperature changes. Especially the $F_{z}$ component was susceptible to large temperature drift due to self heating of the strain gauges. Since this resistive heating affects all gauges equally, the increase in resistance is recorded as apparent force in z-direction. In newer versions the strain gauge configuration was, therefore, changed to a Half-Bridge configuration in order to increase the usable sensor signal and decrease the effects of self heating, effectively decreasing noise, and drift.
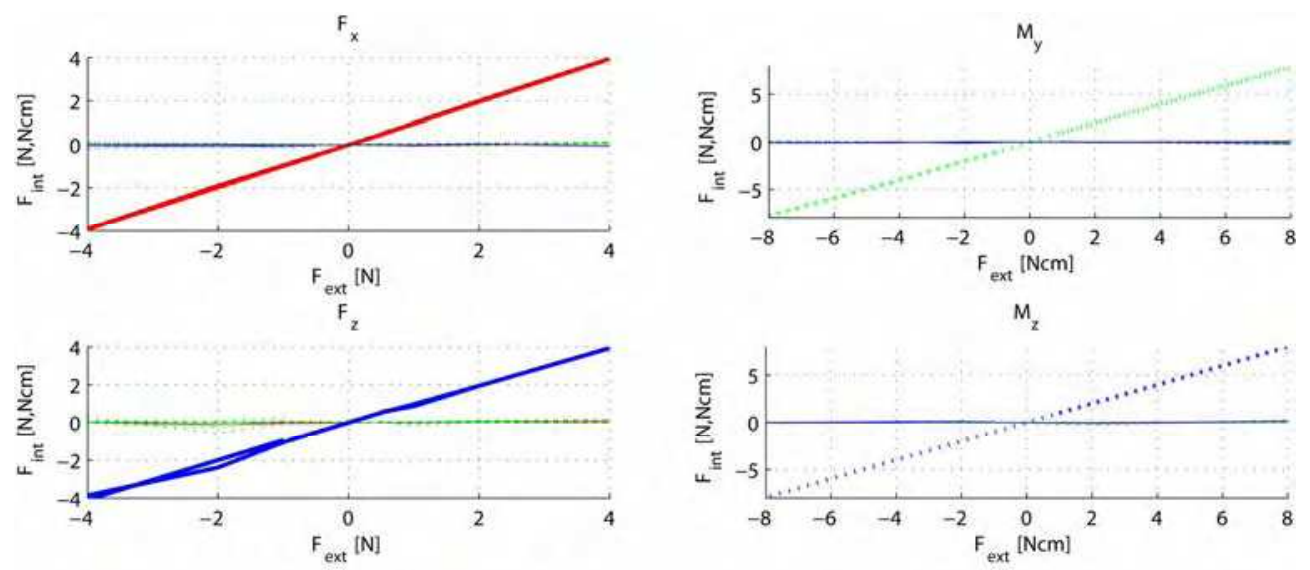

$\mathrm{F}_{x}[\mathrm{~N}] \longrightarrow \mathrm{F}_{y}[\mathrm{~N}]-\mathrm{F}_{z}[\mathrm{~N}] \ldots \ldots \ldots \mathrm{M}_{x}[\mathrm{Nem}] \ldots \ldots \ldots \mathrm{M}_{y}[\mathrm{Ncm}] \ldots \ldots \ldots \mathrm{M}_{z}[\mathrm{Nem}]$

Figure 9. Response of the FTS to externally applied loads

\subsection{Instrument: 2 DoF Wrist}

The length of the distal joint assembly is restricted during surgery by manipulability considerations, as well as the distance between skin and operation site. For abdominal procedures this distance can be increased by insufflation, but due to the rigidity of the ribcage this is not an option for heart procedures. The joint and gripper mechanics should, 
therefore, be kept as short as possible, this is even more important as the sensor increases the length of the distal assembly.

The articulated joint shown in Figure 11 closely resembles a universal joint with intersecting axes, actuated by steel cables. The drive cables in the joint run tangent to the joint pulleys at all times, therefore, the lengths of both cable loops remain constant for every joint position. The middle of each cable loop is fixed at the distal part of the joint, while the proximal ends are connected crosswise at the actuators. With this particular layout only two rotary drives are needed in the propulsion unit to fully actuate the joint, yielding linear transmission characteristics. Driving only one actuator results in a tilting motion of the instrument tip at $45^{\circ}$ angle to the principal axes of the joint:

$$
\begin{aligned}
\theta_{8} & =\frac{r_{M}}{2 r_{a}}(\beta-\alpha), \\
\theta_{9} & =\frac{r_{M}}{2 r_{a}}(\beta+\alpha)
\end{aligned}
$$

with $r_{M}$ : radius of motor pulley, $r_{a}$ : radius of joint pulley $(3 \mathrm{~mm}), \alpha, \beta$ : actuator positions and $\theta_{8}, \theta_{9}$ : joint angles. To guarantee zero backlash, the cables are prestressed with the maximum expected driving force.
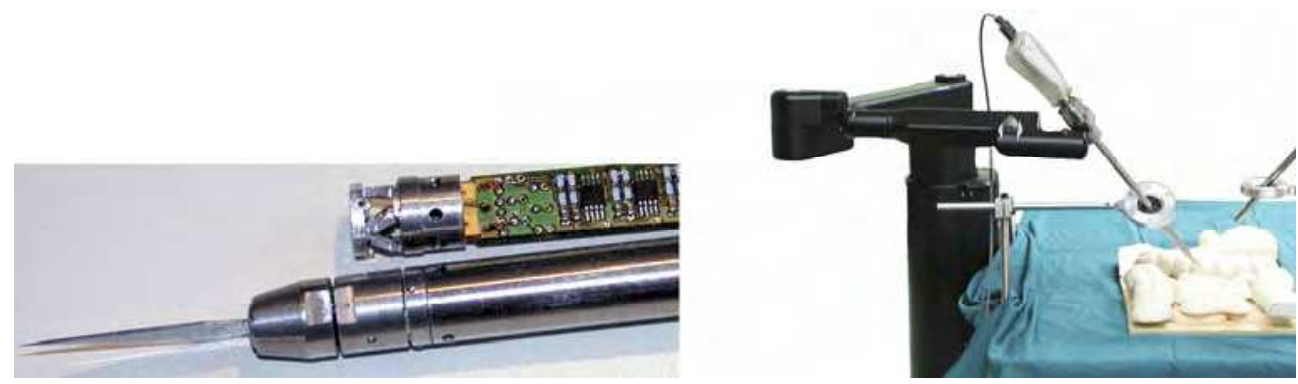

Figure 10. Application of the sensor in an experimental surgical knife, attached to a Zeus manipulator
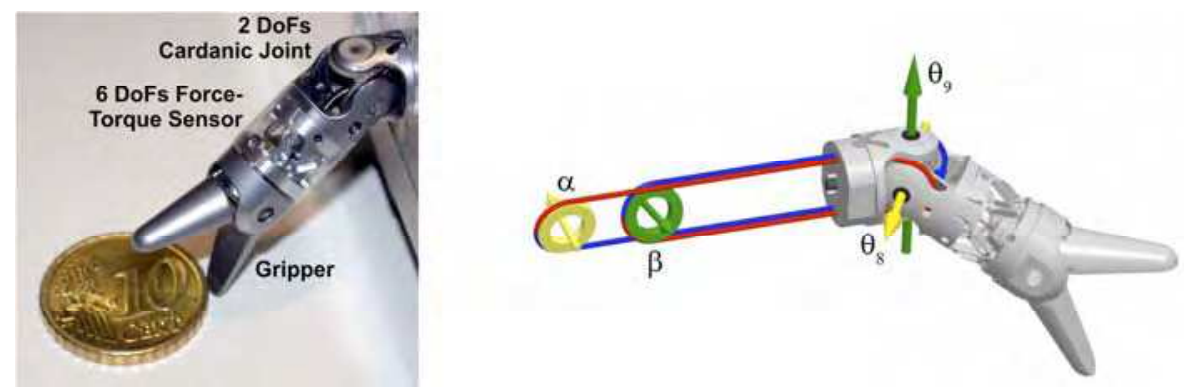

Figure 11. Prototype of instrument tip and layout of the drive cables

\subsection{Haptic Workstation}

To accomplish surgical procedures, the surgeon has to control the instruments, the endoscope, and additional surgical functions (e. g. electrocoagulation, irrigation, suction). The user 
interface of the daVinci system by Intuitive Surgical ${ }^{\circledR}$ is a good example for ergonomics and usability. However, as proprietary system and integral part of the daVinci setup it cannot be used in experimental MIRS systems. A surgeon input console for MIRS commonly includes a (stereo) display and two hand controllers to command the surgical manipulators with up to 7 DoF. Most hand controllers consist of articulated mechanical arms with four to six active and/or passive joints. The position of the surgeon's hands is measured by encoders in every joint. In order to provide a smooth, backlash-free motion these arms consist of a mechanically complicated setup including precisely machined parts and bearings. The console, integrating two arms and a display, is expensive and tends to take up significant space in the operating room. To provide full active force feedback, motors have to be integrated for every joint of the hand controller in addition to the encoders.

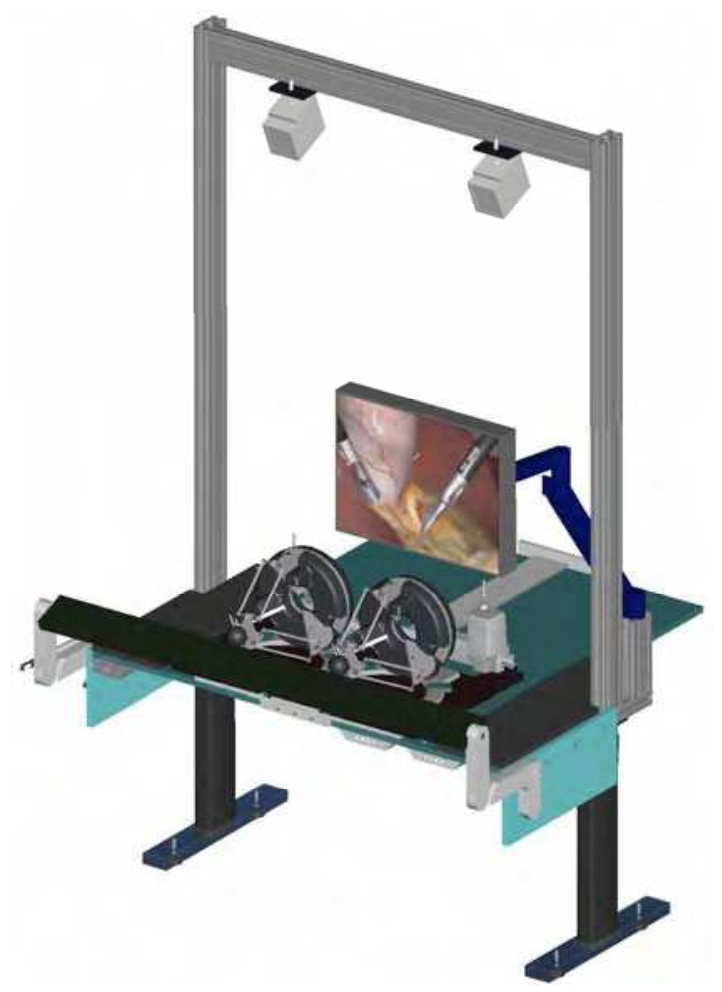

Figure 12. CAD depiction of the proprietary developed telesurgical workstation (master console)

The DLR MIRS workstation will provide intuitive manipulation of up to three instruments, including indexing functions to enlarge the available workspace. Important aspects of this concept are 3-D visualization without the use of shutter glasses or head up displays, haptic feedback, as well as comfortable and strain-free working environment from the 5th percentile female to 95th percentile male. Also, a compact design of the workstation is desired to minimize the spatial requirements in the OR, reduce separation between the 
surgeon and the surgical team, allowing direct visual contact to the anaesthesia area and to ease transportation.

At the current point of the project it is important to provide a generic and open setup, conducive to experimentation with a range of different input and feedback strategies, rather than a finished product.

As haptic input devices commercially available omega.7 devices by ForceDimension are chosen for their reasonable size, sufficient resolution, and high stiffness. The omega.7 device only provides force feedback in $4 \mathrm{DoF}$ ( 3 translations and gripper). At this point, however, no device capable of satisfying all requirements exists. To enhance the precision for intricate tasks, the robotic workstation will provide the possibility of force and motion scaling, generally between $1: 1$ and $1: 10$. The devices are mounted on the workstation facing the surgeon frontally. This way the workspace of the haptic input devices best matches the required workspace for various surgical procedures (Schechner, 2007), however, the handles need to be angled outward to allow for a more natural hand position.

Unlike open surgeries, in MIS the surgeon has no direct visual access to the operation site. Conventionally, an assistant guides the camera, however, this is a strenuous task and requires practice and good collaboration. To compensate for this drawback and to allow for efficient working it is necessary to provide a fast camera control to the surgeon, which can be operated simultaneously to surgical task. As surgeons have emphasized in conversations, the ability to switch between camera positions, such as detail and overview or memory positions, is desired. Since different camera guidance modalities are to be evaluated, camera control is provided with foot pedals, but autonomous camera guidance can also be used (Wei et al., 1997). An auto-stereoscopic LCD display, including eye tracking to adjust for the position of the user, was chosen to provide 3-D visualization.

Comfortable working postures for surgeons of different height require many sections of the workstation to be adjustable. Before determining appropriate ranges, a fixed point of reference was chosen. In airplanes and for microscopic applications the eyes are chosen as fixed point to ensure the best viewing conditions. In cars, generally the fixed point is determined by the position of the foot pedals. For the workstation design, the option of choosing the eyes as fixed point is not reasonable as the view is not determined by a windshield as in cars or airplanes. To the contrary, it is relatively easy to adjust the display according to the posture of the user. The floor is chosen as fixed point, because this allows resting the feet on the ground and also moving the chair with the feet. Furthermore, the surgeon can easily change the position between standing and sitting. Table 3 shows the appropriate adjustment ranges based on ergonomics data.

An armrest is added to the workstation to minimize the load on the shoulder neck area and to allow a strain-free working for the surgeon. The armrest has to be adjustable relatively to the haptic devices in height and depth allowing for support of elbow, forearm, or hand. Failsafe operation of the workstation is of paramount importance. To prevent unintentional operation of the instruments, a pressure sensitive switch is integrated in the armrest to disable movement and force feedback when contact between the surgeon's hands and the armrest is lost.

The generic and open concept of the Surgeon Console described in this section allows for comprehensive experiments, in order to determine the respective impact and benefit of these technologies. 

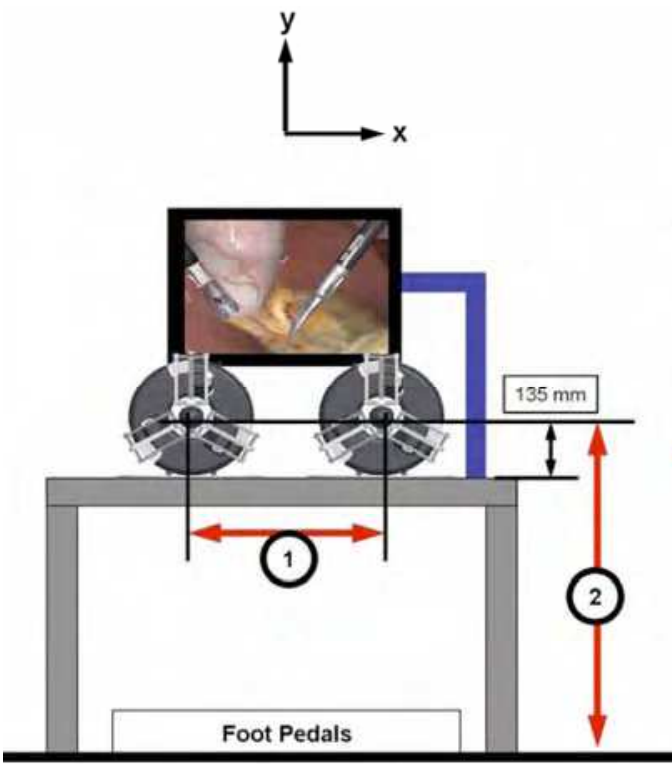
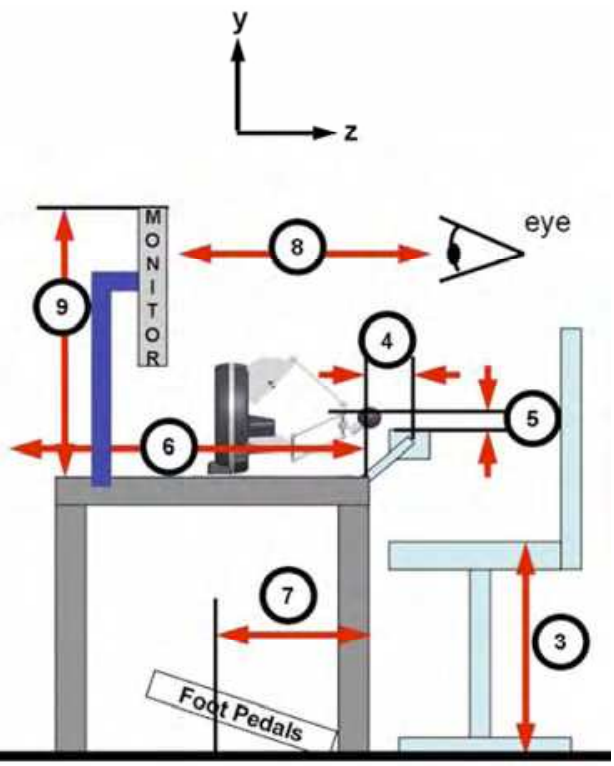

Figure 13. Range of adjustability for the haptic workstation (Schechner, 2007)

\begin{tabular}{|ll|l|}
\hline \multicolumn{2}{|l|}{ Description according to Figure 13 } & Adjustment range \\
\hline 1. & haptic devices relative to each other (x) & $300 \mathrm{~mm}$ to $600 \mathrm{~mm}$ \\
2. & height of the work center (y) & $600 \mathrm{~mm}$ to $1250 \mathrm{~mm}$ \\
3. & height of the chair (y) & $375 \mathrm{~mm}$ to $490 \mathrm{~mm}$ \\
& height of the standing stool (y) & $700 \mathrm{~mm}$ to $850 \mathrm{~mm}$ \\
4. & distance between armrest and device (z) & $60 \mathrm{~mm}$ to $510 \mathrm{~mm}$ \\
5. distance between armrest and device (y) & $-50 \mathrm{~mm}$ to $50 \mathrm{~mm}$ \\
6. & haptic device/optical tracking position (z) & $0 \mathrm{~mm}$ to $600 \mathrm{~mm}$ \\
7. distance between foot pedals and table edge (z) & $250 \mathrm{~mm}$ to $350 \mathrm{~mm}$ \\
8. & distance eye - monitor (z) & $500 \mathrm{~mm}$ to $700 \mathrm{~mm}$ \\
9. & distance monitor - table (y) & $575 \mathrm{~mm}$ to $665 \mathrm{~mm}$ \\
\hline
\end{tabular}

Table 3. Adjustment ranges of the workstation (Schechner, 2007)

\section{Contributions}

In Section 1, three main goals for this project were stated:

1. Demonstrating the feasibility of integrating a force/torque sensor inside an articulated instrument for MIRS.

2. Evaluating the impact and benefit of providing haptic feedback during MIRS.

3. Improving the acceptance of this advanced technology by providing a generic and modular setup applicable to a large number of procedures.

Although the entire system is targeted for minimally invasive as well as for open procedures, minimally invasive cardiac bypass grafting on the beating heart was selected as exemplary application due to the high demands on dynamics, accuracy, and workspace of the manipulator. Two requirements in particular apply to the presented instrument. As 
determined in infeeding tests of surgical needles into cadaveric tissue, intracorporal suturing requires gripping forces of $\sim 10 \mathrm{~N}$ to securely hold a needle and several Newton to securely tie a suture. Furthermore, motion compensation on the beating heart necessitates an actuation frequency of $\sim 3.5 \mathrm{~Hz}$ to follow the motion of the heart surface according to previous research (Ortmaier, 2003). Preliminary experiments showed that specifications could be met with a compact, light weight instrument, consisting of a self contained propulsion unit with integrated motion control and sensor conditioning electronics. An articulated 2 DoF wrist joint and 7 DoF force/torque sensor could be placed inside the distal end of a $10 \mathrm{~mm}$ diameter shaft, thereby, achieving the first goal. With the presented instruments not only pure kinaesthetic feedback can be achieved, also tactile impressions, like rigidification of tissue, can be recognized to some degree: taking tissue between the gripper jaws gives a comparably good impression of rigidity. Surfaces with inconsistent properties can be explored by drawing the tip over the surface with constant contact force; depth of impression will give at least an idea of tissue elasticity. By moving the instrument tip along a surface even coarse textures (surface roughness) can be felt. However, the tactile impressions are not comparable to the sensitivity and resolution of the human skin.

The second goal, assessing the impact and benefit of haptic feedback during surgery, requires the entire system (surgical manipulators, instruments, surgeon workstation, vision system, control hardware) to be assembled and integrated. Only then meaningful experiments can be conducted, comparing the performance of surgeons without haptic feedback and with various forms of feedback at an identical setup. Evaluating the performance of a MIRS force feedback system in an engineering fashion is difficult, since subjective perception (e. g. usability, immersion, user-friendliness) which is not always measurable objectively, plays an important role. Indirect measures like applied forces or time required to complete a defined task can be consulted. With previous hardware a preliminary user study was performed, comparing the dissection of an artery from surrounding tissue using tissue models. It was shown that the surgeon can benefit from high quality haptic feedback. Applied forces were reduced and the procedure was performed more cautiously (Deml et al., 2005), which on the other hand resulted in increased task completion time. It was also shown that high levels of haptic feedback are distracting, whereas low levels provide a more subtle guidance. The hardware is since being improved, and more studies based on tasks required for the proposed application of coronary surgery will need to be performed. Experiments might include tasks comparable to Section 3.1 but also more complex tasks like banding a hollow organ (e. g. a vessel) or performing a suture. The observation of simplified and standardized tasks will lead to better understanding of the whole system performance and future requirements. Additionally, subsequent motion analysis and the recognition of target oriented movements as well as auxiliary motions can be performed. A performance metric has to be found accounting for e.g. the tradeoff between task completion time and error rate. Assembly of the new system is currently in progress.

The third goal can be seen as overall design guide for the system development. Initially, the system will be used for research. It is, therefore, important to ensure modularity so that components can be tested separately, as well as providing generic components that can easily be adapted to the task at hand. This design guide has the added benefit of allowing the system to be used in a number of configurations. Examples include different numbers and arrangements of manipulators, working without MIS instruments for some neurological 
or orthopedic procedures, or employing the system just as autonomous endoscope carrier. The system can also be used as a virtual reality trainer displaying previously recorded procedures to novice surgeons and providing means for learning assessment and quality assurance. This large spectrum of possible applications will help with improved capacity utilization in the OR and, therefore, a better cost benefit ratio. Ensuring the steam sterilizability of all components with direct patient contact falls into the same category, as it allows following the normal clinical workflow.

For the three goals - creating a technology demonstrator, evaluating its impact/benefit, and improving its acceptance - either solutions or research approaches could be demonstrated.

\section{Future Research/Outlook}

The DLR surgical instruments currently allow for one functional DoF which can take shape as gripper, scissors, or clip applicator. The instrument diameter of $10 \mathrm{~mm}$ is in a usual MIS range, but for further improved patient protection as well as for higher manipulability and dexterity in small body cavities, it is desired to further reduce the instrument diameter. Additional functionality like laser application, monopolar electrosurgery in combination with argon plasma coagulation (APC), or ultrasound dissection are standardized methods in surgery which have to be supported. Especially the integration of electrosurgery necessitates the cautery current carrying parts to be insulated from highly sensitive sensor elements, the drive unit with control electronics, and of course tangible parts.

Concerning the everyday use of common surgical robots, instrument changes require a significant portion of the operation time. Reintroducing a new instrument into the body should, for safety reasons, still be guided by an assistant. However, remembering the last instrument position, removing, and switching the instrument could be automated. This would reduce reorientation time and prevent unintended tissue damage during the relocation process.

The presented system is at this point designed as modular research platform rather than for a clinical environment. Therefore, some aspects which are important in a production system (suitability for series production, life-time analysis, etc.) will have to be addressed with the input from experienced industrial partners. Sterilizability was considered, for components with direct patient contact even steam sterilizability, however, in many cases mostly in material selection and not fully in geometrical arrangement. Consequently, a significant amount of work is left in those areas, as well as in refining the "touch and feel" of the system for maximum immersion, necessitating the cooperation with surgeons, industry, and industrial psychologists.

\section{References}

Akinbiyi, T.; Reiley, C.E.; Saha, S.; Burschka, D.; Hasser, C.J.; Yuh, D.D. \& Okamura, A.M. (2006). Dynamic augmented reality for sensory substitution in robot-assisted surgical systems, in: Proceedings of the 28th IEEE EMBS Annual International Conference, pp. 567-570, New York City, NY, USA

Braun, M.; Schurr, M.; Hausam, V. \& Buess, G. (2004). Erweiterte Freiheitsgrade mechanischer Manipulatoren für die minimalinvasive Chirurgie, in: Biomedizinische Technik, vol. 49, pp. 126-127 
Dakin, G. \& Gagner, M. (2003). Comparison of laparoscopic skills performance between standard instruments and two surgical robotic systems, Surgical Endoscopy, vol. 17:pp. 574-579

Demi, B.; Ortmaier, T. \& Seibold, U. (2005). The touch and feel in minimally invasive surgery, in: HAVE 2005 IEEE International Workshop on Haptic Audio Visual Environments and their Applications. Ottawa, Ontario, Canada., pp. 33-38

DLR - German Aerospace Center, Institute of Robotics and Mechatronics (2007). KineMedic Homepage, Web-site, URL http:/ / www.dlr.de/rm-neu/desktopdefault.aspx/tabid-3 828 /

Falk, V.; Diegeler, A.; Walther, T.; Löscher, N.; Vogel, B.; Ulmann, C.; Rauch, T. \& Mohr, F.W. (1999). Endoscopic coronary artery bypass grafting on the beating heart using a computer enhanced telemanipulation system, Heart Surgery Forum, vol. 2:pp. 199-205

Frede, T.; Hammadya, A.; Klein, J.; Teber, D.; Inaki, N.; Waseda, M.; Buess, G. \& Rassweiler, J. (2007). The radius surgical system - a new device for complex minimally invasive procedures in urology?, European Urology, vol. 51:pp. 1015-1022

Guthart, G. \& Salisbury, J. (2000). The intuitive telesurgery system: Overview and application, in: Procedings of the IEEE International Conference on Robotics and Automation (ICRA), vol. 1, pp. 618-621, San Francisco, CA, USA, ISBN 0-7803-5886-4

Hernandez, J.; Barm, S.; Munz, Y.; Moorthy, K.; Datta, V.; Martin, S.; Dosis, A.; Bello, F.; Darzi, A. \& Rockall, T. (2004). Qualitative and quantitative analysis of the learning curve of a simulated surgical task on the daVinci system, Surgical Endoscopy, vol. 18:pp. 372-378

Inaki, N.; Waseda, M.; Schurr, M.; Braun, M. \& Buess, G. (2007). Experimental results of mesh fixation by a manual manipulator in a laparoscopic inguinal hernia repair model, Surgical Endoscopy, vol. 21(2):pp. 197-201

Intuitive Surgical, Inc. (2007). Company profile of Intuitive Surgical, Inc., Sunnyvale, CA, USA, Website, URL http:/ / www.intuitivesurgical.com

Kitagawa, M.; Dokko, D.; Okamura, A.M.; Bethea, B.T. \& Yuh, D.D. (2004). Effect of sensory substitution on suture manipulation forces for surgical teleoperation, in: Annual Medicine Meets Virtual Reality Conference (MMVR), Newport Beach, CA, USA, submitted

Kitagawa, M. (2003). Indirect Feedback of Haptic Information for Robot-Assisted Telemanipulation, Master's thesis, Johns Hopkins University

Konietschke, R.; Ortmaier, T.; Weiss, H.; Engelke, R. \& Hirzinger, G. (2003). Force propagation models in laparoscopic tools and trainers, in: 2nd Conference of the German Society of Computer and Robotic Assisted Surgery (CURAC), Nürnberg, Germany

Konietschke, R.; Ortmaier, T.; Weiss, H.; Hirzinger, G. \& Engelke, R. (2004). Manipulability and accuracy measures for a medical robot in minimally invasive surgery, in: 9 th International Symposium on Advances in Robot Kinematics

Kwon, D.S.; Woo, K.Y.; Song, S.K.; Kim, W.S. \& Cho, H.S. (1998). Microsurgical telerobot system, in: Proceedings of the IEEE/RSJ International Conference on Intelligent Robots and Systems (IROS), pp. 945-950

Lobontiu, N. (2002). Compliant Mechanisms: Design of Flexure Hinges, CRC Press Inc., ISBN-10: 0849313678, ISBN-13: 978-0849313677 
MacFarlane, M.; Rosen, J.; Hannaford, B.; Pellegrini, C. \& Sinanan, M. (1999). Force-feedback grasper helps restore sense of touch in minimally invasive surgery, Journal of Gastrointestinal Surgery, vol. 3(3):pp. 278-285

Mayer, H.; Nagy, I.; Knoll, A.; Schirmbeck, E. \& Bauernschmitt, R. (2004). Upgrading instruments for robotic surgery, in: Australasian Conference on Robotics $\mathcal{E}$ Automation

Müller, J. (2004). Charite, Klinik für Allgemein-, Visceral-, Gefäß- und Thoraxchirurgie, Campus Mitte, from discussion, Berlin, Germany

Nakamura, Y. (2003). Virtual stillness and small size robot system that occupies less space in OR, in: Proceedings of the IEEE International Conference on Robotics and Automation (ICRA), Workshop Recent Advances in Medical Robotics, Taipei, Taiwan

Omote, K.; Feussner, H.; Ungeheuer, A.; Arbter, K.; Wei, G.Q.; Siewert, J.R. \& Hirzinger, G. (1999). Self-guided robotic camera control for laparoscopic surgery compared with human camera control, The American Journal of Surgery, vol. 117:pp. 321-324

Ortmaier, T.; Weiss, H. \& Hirzinger, G. (2003). Minimally invasive robotic surgery: Foundations and perspectives, in: Proceedings of the IEEE International Conference on Robotics and Automation (ICRA), Workshop on Recent Advances in Medical Robotics, Taipei, Taiwan

Ortmaier, T.; Weiss, H.; Hagn, U.; Grebenstein, M.; Nickl, M.; Albu-Schäffer, A.; Ott, C.; Jörg, S.; Konietschke, R.; Le-Tien, L. \& Hirzinger, G. (2006). A hands-on-robot for accurate placement of pedicle screws, in: Proceedings of the IEEE International Conference on Robotics and Automation (ICRA), Orlando, Florida, USA

Ortmaier, T. (2003). Motion Compensation in Minimally Invasive Robotic Surgery, VDI Verlag, $\mathrm{PhD}$ Thesis

Peirs, J.; Clijnen, J.; Reynaerts, D.; Brussel, H.V.; Herijgers, P.; Corteville, B. \& Boone, S. (2004). A micro optical force sensor for force feedback during minimally invasive robotic surgery, Sensors and Actuators A, vol. 115:pp. 447-455

Rosen, J. (2001). Human machine interfaces in minimally invasive surgery, in: Medicine Meets Virtual Reality (MMVR), Workshop on Simulating Minimally Invasive Surgical Procedures in Virtual Environments

Rosen, J.; Hannaford, B.; MacFarlane, M. \& Sinanan, M. (1999a). Force controlled and teleoperated endoscopic grasper for minimally invasive surgery - experimental performance evaluation, IEEE Transactions on Biomedical Engineering, vol. 46(10)

Rosen, J.; MacFarlane, M.; Richards, C.; Hannaford, B. \& Sinanan, M. (1999b). Surgeon-tool force/torque signatures - evaluation of surgical skills in minimally invasive surgery, in: Annual Medicine Meets Virtual Reality Conference (MMVR), vol. 7

Salle, D.; Bidaud, P. \& Morel, G. (2004). Optimal design of high dexterity modular MIS instrument for coronary artery bypass grafting, in: Procedings of the IEEE International Conference on Robotics and Automation (ICRA), vol. 2, pp. 1276-1281, ISSN: 1050-4729, ISBN: 0-7803-8232-3

Schechner, V. (2007). Concept and Ergonomics of a Bi-Manual Haptic Workstation for Minimally Invasive Robotic Surgery, Master's thesis, Lehrstuhl für Ergonomie, Technische Universität München, Munich, Germany

Seibold, U. (2002). Monolithic Force-Torque Sensor (Kraft-Moment-Sensor), German Patent 10217018.5 
Seibold, U. \& Hirzinger, G. (2003). A 6-axis force/torque sensor design for haptic feedback in minimally invasive robotic surgery, in: MICRO.tec - 2nd VDE World Microtechnologies Congress, pp. 239-244

Sorli, M. \& Pastorelli, S. (1995). Six-axis reticulated structure force/torque sensor with adaptable performances, Mechatronics, vol. 5(6):pp. 585-601

Tendick, F. \& Cenk Cavusoglu, M. (1997). Human-Machine Interfaces for Minimally Invasive Surgery, Proceedings of the 19th Annual International Conference of the IEEE Engineering in Medicine and Biology Society, pp. 2771-2776, Chicago, IL, USA

Tholey, G.; Desai, J.P. \& Castellanos, A.E. (2003). Evaluating the role of vision and force feedback in minimally invasive surgery: New automated laparoscopic grasper and a case study, in: Proceeding of the Medical Image Computing and Computer-Assisted Intervention (MICCAI)

Tholey, G.; Pillarisetti, A.; Green, W. \& Desai, J.P. (2004). Design, Development, and Testing of an Automated Laparoscopic Grasper with 3-D Force Measurement Capability, vol. 3078, Springer Berlin / Heidelberg

Tuebingen Scientific Medical, G. (2007). Homepage of Tuebingen Scientific Medical, Inc., Tuebingen, Germany, Website, URL http://www.tuebingen-scientific.de

Wagner, C.; Stylopoulos, N. \& Howe, R. (2002). The role of force feedback in surgery: Analysis of blunt dissection, in: Proceedings of the 10th Symposium on Haptic Interfaces for Virtual Environment and Teleoperator Systems (HAPTICS), pp. 68-74, ISBN: 0-76951489-8

Wei, G.; Arbter, K. \& Hirzinger, G. (1997). Real-time visual servoing for laparoscopic surgery, IEEE Engineering in Medicine and Biology, vol. 16(1)

Zemiti, N.; Ortmaier, T.; Vitrani, M. \& Morel, G. (2004). A force controlled laparoscopic surgical robot without distal force sensing, in: Proceedings of the International Symposium on Experimental Robotics (ISER) 


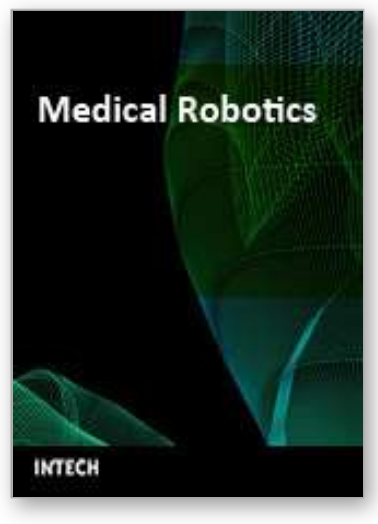

\author{
Medical Robotics \\ Edited by Vanja Bozovic
}

ISBN 978-3-902613-18-9

Hard cover, 526 pages

Publisher I-Tech Education and Publishing

Published online 01, January, 2008

Published in print edition January, 2008

The first generation of surgical robots are already being installed in a number of operating rooms around the world. Robotics is being introduced to medicine because it allows for unprecedented control and precision of surgical instruments in minimally invasive procedures. So far, robots have been used to position an endoscope, perform gallbladder surgery and correct gastroesophogeal reflux and heartburn. The ultimate goal of the robotic surgery field is to design a robot that can be used to perform closed-chest, beating-heart surgery. The use of robotics in surgery will expand over the next decades without any doubt. Minimally Invasive Surgery (MIS) is a revolutionary approach in surgery. In MIS, the operation is performed with instruments and viewing equipment inserted into the body through small incisions created by the surgeon, in contrast to open surgery with large incisions. This minimizes surgical trauma and damage to healthy tissue, resulting in shorter patient recovery time. The aim of this book is to provide an overview of the state-of-art, to present new ideas, original results and practical experiences in this expanding area. Nevertheless, many chapters in the book concern advanced research on this growing area. The book provides critical analysis of clinical trials, assessment of the benefits and risks of the application of these technologies. This book is certainly a small sample of the research activity on Medical Robotics going on around the globe as you read it, but it surely covers a good deal of what has been done in the field recently, and as such it works as a valuable source for researchers interested in the involved subjects, whether they are currently "medical roboticists" or not.

\title{
How to reference
}

In order to correctly reference this scholarly work, feel free to copy and paste the following:

Ulrich Seibold, Bernhard Kuebler and Gerd Hirzinger (2008). Prototypic Force Feedback Instrument for Minimally Invasive Robotic Surgery, Medical Robotics, Vanja Bozovic (Ed.), ISBN: 978-3-902613-18-9, InTech, Available from:

http://www.intechopen.com/books/medical_robotics/prototypic_force_feedback_instrument_for_minimally_inva sive_robotic_surgery

\section{INTECH}

open science | open minds

\section{InTech Europe}

University Campus STeP Ri

Slavka Krautzeka 83/A

\section{InTech China}

Unit 405, Office Block, Hotel Equatorial Shanghai

No.65, Yan An Road (West), Shanghai, 200040, China 
51000 Rijeka, Croatia

Phone: +385 (51) 770447

Fax: +385 (51) 686166

www.intechopen.com
中国上海市延安西路65号上海国际贵都大饭店办公楼 405 单元 Phone: +86-21-62489820

Fax: +86-21-62489821 
(C) 2008 The Author(s). Licensee IntechOpen. This chapter is distributed under the terms of the Creative Commons Attribution-NonCommercialShareAlike-3.0 License, which permits use, distribution and reproduction for non-commercial purposes, provided the original is properly cited and derivative works building on this content are distributed under the same license. 